\title{
Analysis on Wireless Power Transmission using different Electromagnetic Fields
}

\author{
A. B. M. Khalid \\ Hassan \\ EEE Department \\ American International \\ University-Bangladesh \\ Dhaka, Bangladesh
}

\author{
Abdullahil Fawaz \\ EEE Department \\ American International \\ University-Bangladesh \\ Dhaka, Bangladesh
}

\author{
Md. Rokibul Hassan \\ Billah \\ EEE Department \\ American International \\ University-Bangladesh \\ Dhaka, Bangladesh
}

\author{
Md. Imran Khan \\ EEE Department \\ American International \\ University-Bangladesh \\ Dhaka, Bangladesh
}

\begin{abstract}
In this paper, the analysis for data collected for wireless power transmission modules through both alternating magnetic field and discontinuous direct electromagnetic field are presented. The comparison of wireless power transmission range, total transferred power as well as efficiency between two different wireless power transmission modules where, one is using alternating electromagnetic field and another one is using discontinuous direct electromagnetic field to transfer electrical power wirelessly, are presented keeping the transmitter coil and receiver coil identical as well as keeping applied voltage same in both cases. All the collected data are presented in both tabular format and graphical format. The input and output waveforms in case of both electromagnetic fields are also been analyzed in this paper.
\end{abstract}

\section{Keywords}

Efficiency, Transmission Range, Total Transferred Power, Wireless Power

\section{INTRODUCTION}

To transfer electricity wirelessly, alternating electromagnetic field is not essential. Electricity can be transmitted wirelessly by using discontinuous direct electromagnetic field [1]. To create an alternating electromagnetic field, $\mathrm{AC}$ power needs to be provided to the transmitter coil and to create a discontinuous direct electromagnetic field, discrete DC power needs to be provided to the transmitter coil. When an alternating electromagnetic field will be received by a receiver coil, an AC signal will be received at the receiver end. Then it is possible to convert that $\mathrm{AC}$ signal into a DC signal to supply a DC load. In case of discontinuous direct electromagnetic field, some DC pulses will be received at the receiver end [1]. Then a linear DC signal is possible to found from that DC pulses to supply a DC load. In both cases, the received voltage, current or total transferred power varies with the distance between transmitter and receiver. By observing those parameters in different transmitter receiver distances for both electromagnetic field, the efficiency could be possible to compare.

For simplicity, all the circuitry have been reduced in this process. Only two identical inductor coils have been used as transmitter and receiver. To generate an alternating electromagnetic field, a sinusoidal AC signal has been provided to the transmitter coil, which has been presented in "Fig. 1," and to generate a discontinuous direct electromagnetic field, a discrete DC signal has been provided to the transmitter coil,

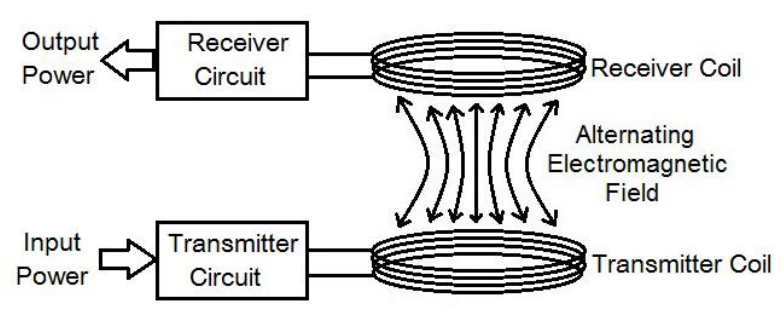

Fig. 1. Measuring total wirelessly transferred electrical power through alternating electromagnetic field.

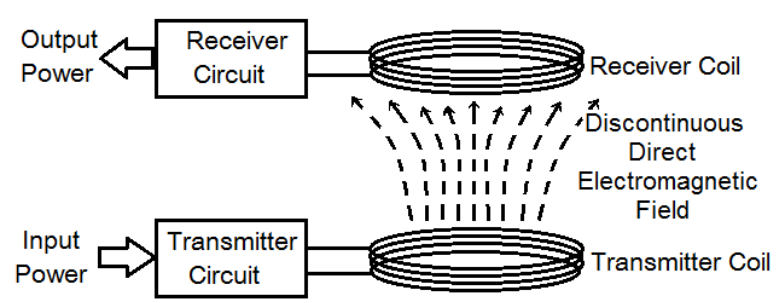

Fig. 2. Measuring total wirelessly transferred electrical power through discontinuous direct electromagnetic field.

which has been presented in "Fig. 2." The output signals have been observed by connecting an oscilloscope directly across the receiver coil in both cases. Mechanical switching has been applied to generate a discrete DC signal. No resonance capacitor has been applied in parallel with inductor coils in any case. This methodology has been followed to get the clear observation of input and output wave signals in both cases.

\section{WAVEFORM OBSERVATIONS}

Fig. 3. To generate an alternating electromagnetic field, an alternating signal has been applied to the transmitter coil, which has been presented in "Fig. 3," and to generate a discontinuous direct electromagnetic field, a discrete DC signal has been applied to the transmission coil, which has been presented in "Fig. 4." The output wave signal for both cases have also been presented in "Fig. 3" and "Fig. 4."

The wave shape of receiver coil tends to be half sinusoidal pulse for alternating electromagnetic field, and half triangular pulse for discontinuous direct electromagnetic field. 


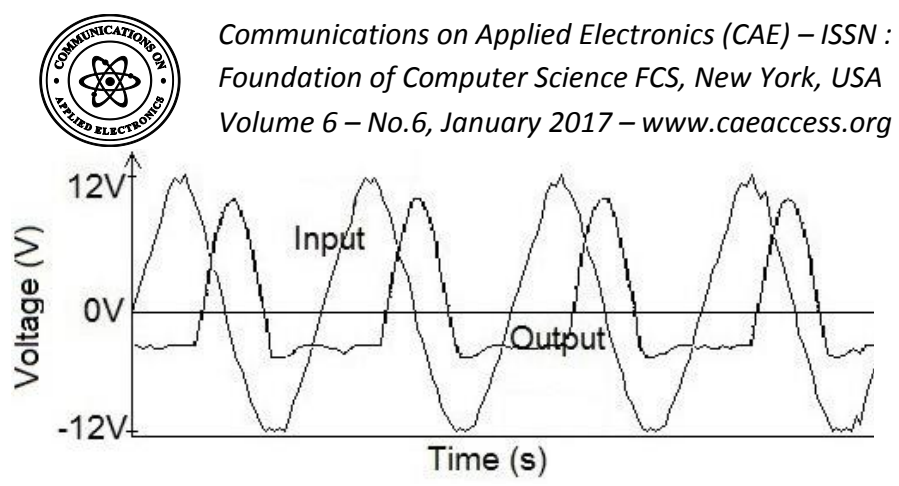

Fig. 3. Experimental wave shapes for alternating electromagnetic field.

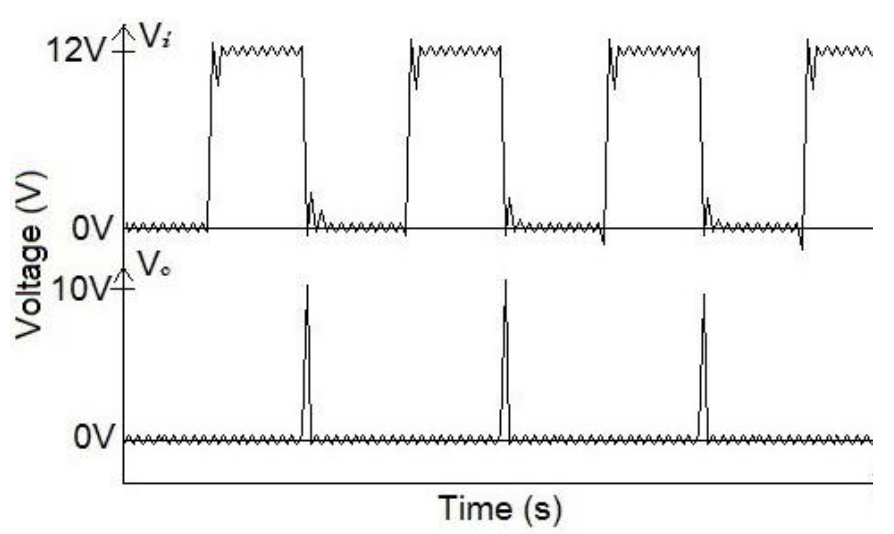

Fig. 4. Experimental wave shapes for discontinuous direct electromagnetic field.

Comparing output wave shapes of both cases, it can be seen that, in alternating electromagnetic field, the output pulses which have been received by the receiver coil, are wider than the output pulses which have been received by the receiver coil in discontinuous direct electromagnetic field. As well as, all the peaks of the output wave are at similar height. On the other hand, in discontinuous direct electromagnetic field, the output pulses which have been received by the receiver coil, are narrow in width and at peaks of the output wave signal are not similar in height. "Fig. 5" is the output wave signal of receiver coil inside a discontinuous direct electromagnetic field, which shows that, all the peaks of the output wave signal are in different heights.

In both cases, operating frequency can be increased to get more cycles in the same time to transfer more energy wirelessly at the same time. But, from those DC pulses of "Fig. 5", a linear DC voltage can be easier to achieve than the other one. But, the received AC signal of "Fig. 3" will need to be converted at every frequency to get a linear DC signal. "Fig. 4" indicates that, the net power loss per cycle will be decreased with shorter pulse widths. That means, the net power loss per cycle will decreased at high frequency, as the duty cycle will be increases with the increase of frequency. "Fig. 3" indicates that, the net power loss will also be decreased with high frequency, but the decreasing will be slower with respect to "Fig. 4" as the duty cycle will not increase with the increase of frequency.

\section{VOLTAGE AND CURRENT OBSERVATIONS}

In every cases of voltage observation, the RMS value has been calculated. To create an alternating magnetic field, a sinusoidal AC voltage has been provided to the transmitter coil where the peak voltage was $12 \mathrm{~V}$. So, the RMS value will be,

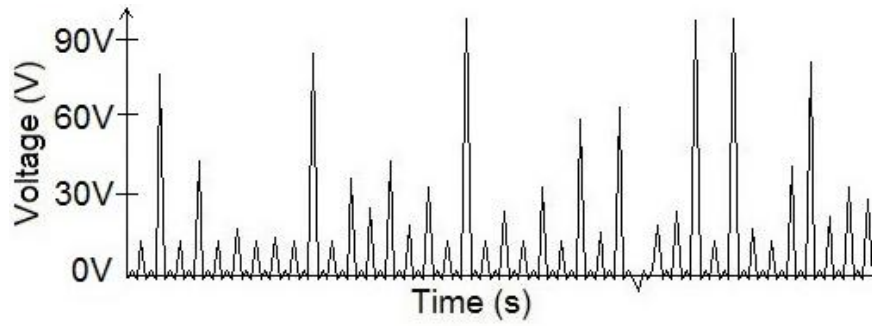

Fig. 5. Experimental output wave shape of discontinuous direct electromagnetic field.

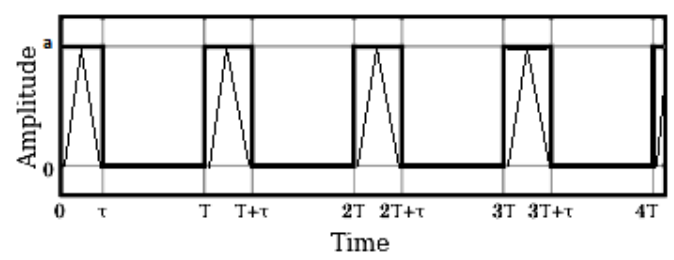

Fig. 6. RMS value calculation for triangular pulse train.

$$
V_{R M S}=\frac{12}{\sqrt{2}}=8.49
$$

The output RMS voltage, which has been received by the receiver coil inside an alternating electromagnetic field, depends on the distance between the transmitter coil and the receiver coil. It has been observed by using a calibrated true RMS multi meter, which have been presented in "Table 1" for different distances between the transmitter coil and the receiver coil. In this case, the operating frequency was comparatively very high as $127 \mathrm{kHz}$. To create a discontinuous direct electromagnetic field, a discrete DC voltage has been provided to the transmitter coil where the peak voltage was $12 \mathrm{~V}$ and the duty cycle was $50 \%$. So, the RMS value will be,

$$
V_{R M S}=12 \sqrt{0.5}=8.49
$$

The output RMS voltage, which has been received by the receiver coil inside a discontinuous direct electromagnetic field, also depends on the distance between the transmitter coil and the receiver coil. The output voltage signal (Fig.5) is a triangular pulse train. For, a rectangular pulse train, the RMS value can be calculated by using the equation,

$$
V_{R M S}=a \sqrt{D}
$$

Where, $\mathrm{a}=$ amplitude and $\mathrm{D}=$ duty cycle of the signal. "Fig. 6 " shows that, the RMS value of a triangular pulse train will be half of the RMS value of a rectangular pulse train. So, the RMS value of that signal has been calculated by using the equation,

$$
V_{R M S}=\frac{a}{2} \sqrt{D}
$$

Where,

$$
D=\frac{\tau}{T}
$$

Here, $\tau=$ pulse width of the rectangular pulse train and $\mathrm{T}=$ cycle length in time axis. It have also been presented in "Table 1" for different distances between the transmitter coil 
and the receiver coil. In this case, the operating frequency was very low as $10 \mathrm{~Hz}$.

Table 1. Output voltages of both cases at different distances.

\begin{tabular}{|c|c|c|c|}
\hline $\begin{array}{c}\text { Distance } \\
(\mathbf{c m})\end{array}$ & $\begin{array}{c}\text { Alternating } \\
\text { EM Field } \\
\left(\mathbf{V}_{\text {RMS }}\right)\end{array}$ & $\begin{array}{c}\text { DDEM } \\
\text { Field at } \\
\text { Lower } \\
\text { Frequency } \\
\left(\mathbf{V}_{\text {RMS }}\right)\end{array}$ & $\begin{array}{c}\text { DDEM } \\
\text { Field at } \\
\text { Higher } \\
\text { Frequency } \\
\left(\mathbf{V}_{\text {RMS }}\right)\end{array}$ \\
\hline 0 & 27.57 & 6.16 & 19.6 \\
\hline 1 & 11.31 & 2.97 & 9.45 \\
\hline 2 & 5.66 & 1.21 & 3.85 \\
\hline 3 & 3.54 & 0.77 & 2.45 \\
\hline 5 & 1.98 & 0.55 & 1.75 \\
\hline 5 & 0.85 & 0.33 & 1.05 \\
\hline
\end{tabular}

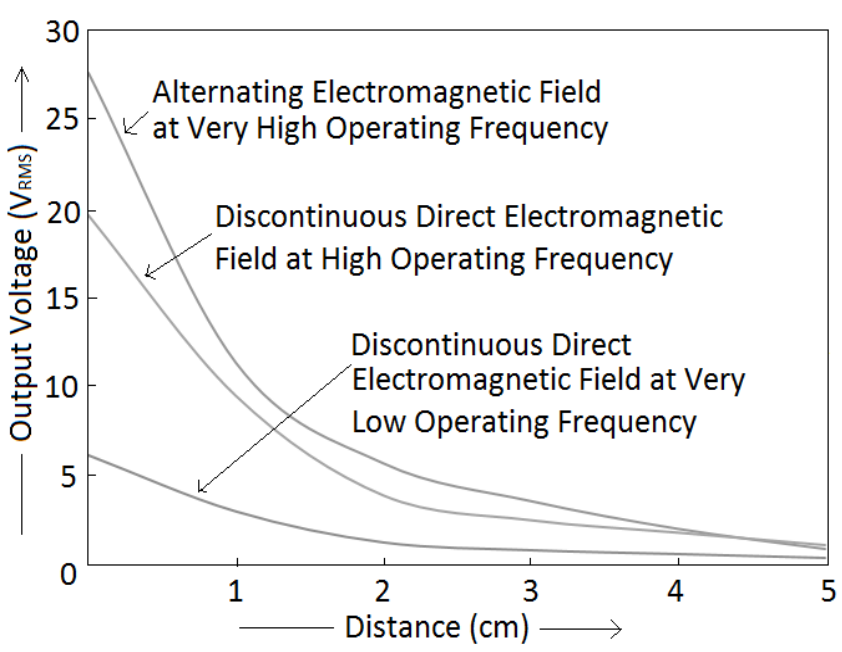

Fig. 7. Graphical representation of output voltages of both cases at different distances.

Comparing the wirelessly received output voltages which have been presented in "Table 1" in case of both alternating electromagnetic field and discontinuous direct electromagnetic field, it can be seen that, the wirelessly received output voltage in case of discontinuous direct electromagnetic field is always smaller than the wirelessly received output voltage in case ofalternating electromagnetic field at each transmitter-receiver distance at lower operating frequencies as $10 \mathrm{~Hz}$. The reason of that is, the duty cycle of the wirelessly received output voltage in case of discontinuous direct electromagnetic field is very low as $5 \%$ at lower operating frequency as $10 \mathrm{~Hz}$. If the duty cycle can be increased to $50 \%$ by increasing the operating frequency, "Fig. 7" presents that, the graph will reach close to the curve of alternating electromagnetic field and before reaching $5 \mathrm{~cm}$ distance between transmitter coil and receiver coil, the graphs crosses one another. That means, the performance of discontinuous direct electromagnetic field at high operating frequency will be better with the increase of distance between the transmitter coil and the receiver coil with respect to alternating electromagnetic field for wireless power transmission. From the graph of "Fig. 7" it can be observed that, to operate a 5VDC load from received wirelessly received power, the maximum transmitter receiver distance will be $2.2 \mathrm{~cm}$ in case of alternating electromagnetic field at very high operating frequency, $1.7 \mathrm{~cm}$ in case of discontinuous direct electromagnetic field at high operating frequency and $0.4 \mathrm{~cm}$ in case of discontinuous direct electromagnetic field at very low operating frequency.

Table 2. Input and output currents in case of alternating electromagnetic field at different distances.

\begin{tabular}{|c|c|c|}
\hline $\begin{array}{c}\text { Distance } \\
(\mathbf{c m})\end{array}$ & Input $\mathbf{I}_{\mathbf{R M S}}$ & Output $\mathbf{I}_{\mathbf{R M S}}$ \\
\hline 0 & 1.01 & 0.31 \\
\hline 1 & 0.82 & 0.29 \\
\hline 2 & 0.66 & 0.16 \\
\hline 3 & 0.48 & 0.13 \\
\hline 4 & 0.20 & 0.09 \\
\hline 5 & 0.09 & 0.06 \\
\hline
\end{tabular}

All the current signals have been observed as same as voltage signal. So, it has been measured in the same way as the voltage signal. In case of alternating electromagnetic field, the RMS value of both input currents and output currents depend on the distance between the transmitter coil and the receiver coil, which has been presented in "Table 2". It presents that, both input current and output current decreases with the increase of distance between transmitter coil and the receiver coil.

Table 3. Input and output currents in case of discontinuous direct electromagnetic field at different distances.

\begin{tabular}{|c|c|c|}
\hline $\begin{array}{c}\text { Distance } \\
(\mathbf{c m})\end{array}$ & Input $\mathbf{I}_{\mathbf{R M S}}$ & Output $\mathbf{I}_{\mathbf{R M S}}$ \\
\hline 0 & 1.41 & 1.39 \\
\hline 1 & 1.41 & 1.16 \\
\hline 2 & 1.41 & 0.98 \\
\hline 3 & 1.41 & 0.79 \\
\hline 4 & 1.41 & 0.53 \\
\hline 5 & 1.41 & 0.28 \\
\hline
\end{tabular}

In case of discontinuous direct electromagnetic field, peak current of input current signal was approximately $2 \mathrm{~A}$ at each 
transmitter receiver distance. So, the RMS value of input current was,

$$
I_{R M S}=2 \sqrt{0.5}=1.41 \mathrm{~A}
$$

The output current depends on the distance between transmitter coil and receiver coil, which has been presented in "Table 3". "Table 3" presents that, the input current is always same but the output current decreases with the increase of distance between transmitter coil and the receiver coil.

\section{POWER AND EFFICIENCY \\ CALCULATION}

The total input power as well as output power has been calculated using this equation,

$$
P_{T O T A L}=V_{R M S} \times I_{R M S}
$$

The input and output power in case of alternating electromagnetic field has been presented in "Table 4". It presents that, both input and output power decreases with the increase of distance between the transmitter coil and the receiver coil.

Table 4. Input and output power in case of alternating electromagnetic field at different distances.

\begin{tabular}{|c|c|c|}
\hline $\begin{array}{c}\text { Distance } \\
(\mathbf{c m})\end{array}$ & $\begin{array}{c}\text { Input Power } \\
(\mathbf{W})\end{array}$ & $\begin{array}{c}\text { Output Power } \\
(\mathbf{W})\end{array}$ \\
\hline 0 & 8.57 & 8.55 \\
\hline 1 & 6.96 & 3.27 \\
\hline 2 & 5.60 & 0.91 \\
\hline 3 & 4.08 & 0.46 \\
\hline 4 & 1.70 & 0.18 \\
\hline 5 & 0.76 & 0.05 \\
\hline
\end{tabular}

The input and output power in case of discontinuous direct electromagnetic field has been presented in "Table 5". It presents that, the input power remains same at each transmitter receiver distance but the output power decreases with the increase of distance between the transmitter coil and the receiver coil.

Table 5. Input and output power in case of discontinuous direct electromagnetic field at different distances.

\begin{tabular}{|c|c|c|}
\hline $\begin{array}{c}\text { Distance } \\
(\mathbf{c m})\end{array}$ & $\begin{array}{c}\text { Input Power } \\
(\mathbf{W})\end{array}$ & $\begin{array}{c}\text { Output Power } \\
(\mathbf{W})\end{array}$ \\
\hline 0 & 11.97 & 8.56 \\
\hline 1 & 11.97 & 3.44 \\
\hline 2 & 11.97 & 1.19 \\
\hline 3 & 11.97 & 0.61 \\
\hline
\end{tabular}

\begin{tabular}{|c|c|c|}
\hline 4 & 11.97 & 0.29 \\
\hline 5 & 11.97 & 0.09 \\
\hline
\end{tabular}

"Fig. 8" represents the comparison of wirelessly received total output power between alternating electromagnetic field and

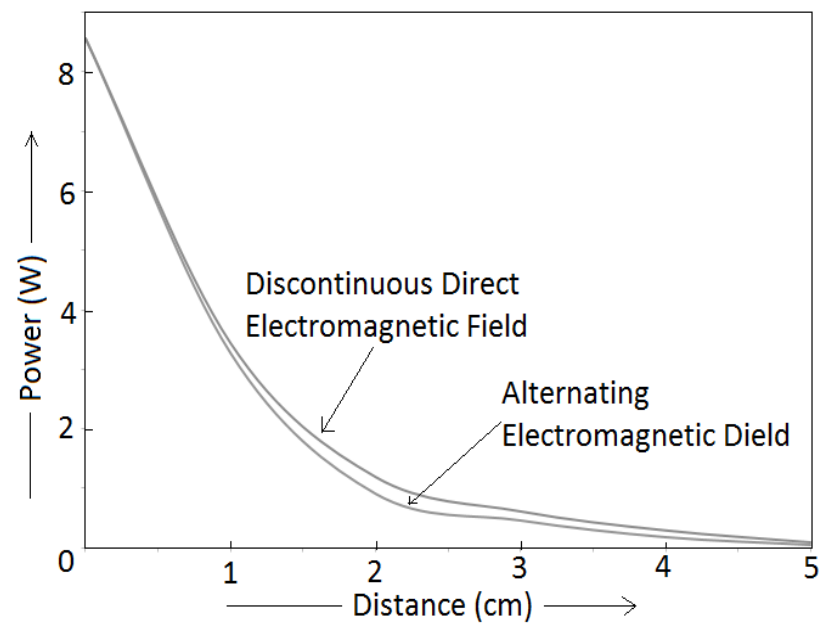

Fig. 8. Graphical representation of output power of both cases at different distances.

discontinuous direct electromagnetic field. It presents that, the total wirelessly received power in case of discontinuous direct electromagnetic field is slightly greater than the total wirelessly received power in case of alternating electromagnetic field at most of the distances in between $0 \mathrm{~cm}$ to $5 \mathrm{~cm}$.

The net amount of power loss for both cases has been presented in "Table 6". In case of discontinuous direct electromagnetic field, as the input power remains same at each transmitter receiver distances, the net amount of power loss increases with the increase of distance between the transmitter coil and the receiver coil. The net amount of power loss has been calculated using this equation,

$$
P_{\text {LOSS }}=P_{\text {INPUT }}-P_{\text {OUTPUT }}
$$

Table 6. Net power loss of both cases at different distances.

\begin{tabular}{|c|c|c|}
\hline $\begin{array}{c}\text { Distance } \\
(\mathbf{c m})\end{array}$ & $\begin{array}{c}\text { Alternating } \\
\text { EM Field } \\
(\mathbf{W})\end{array}$ & $\begin{array}{c}\text { DDEM Field } \\
(\mathbf{W})\end{array}$ \\
\hline 0 & 0.02 & 3.41 \\
\hline 1 & 3.69 & 8.53 \\
\hline 2 & 4.69 & 10.78 \\
\hline 3 & 3.62 & 11.36 \\
\hline 4 & 1.52 & 11.68 \\
\hline 5 & 0.71 & 11.88 \\
\hline
\end{tabular}

The data presented in "Table 6" has been plotted in "Fig. 9" which presents that, in case of alternating electromagnetic field, the net amount of power loss is very low as $0.02 \mathrm{~W}$ at 
$0 \mathrm{~cm}$ distance between the transmitter coil and the receiver coil. Then, it has been increased up to $4.69 \mathrm{~W}$ at $2 \mathrm{~cm}$ distance between the transmitter coil and the receiver coil. Then, it has been decrease again to $0.71 \mathrm{~W}$ at $5 \mathrm{~cm}$ distance between the

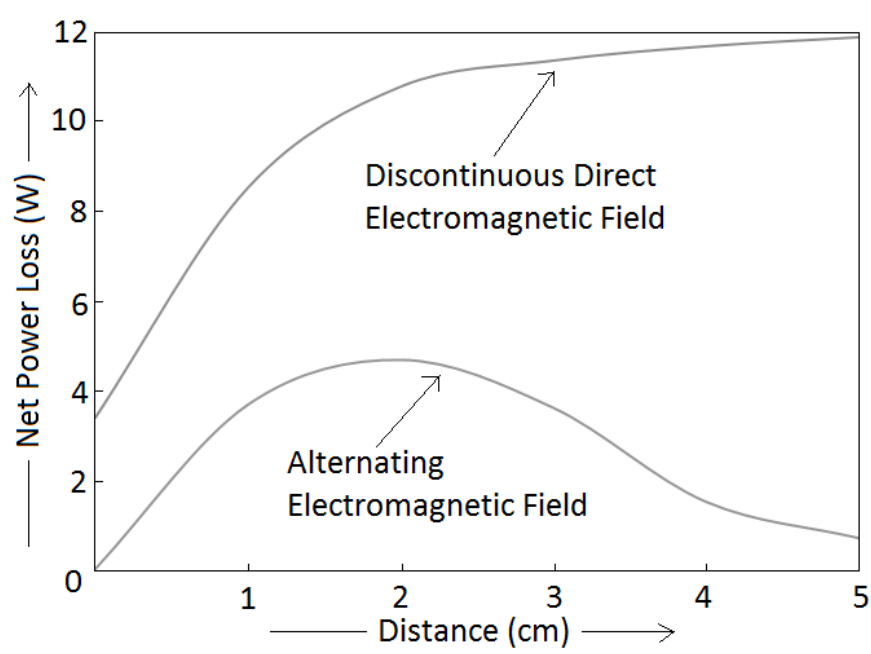

Fig. 9. Graphical representation of net power loss of both cases at different distances.

transmitter coil and the receiver coil. In case of discontinuous direct electromagnetic field, as the total input power remains high as $11.97 \mathrm{~W}$ at each distance between the transmitter coil and the receiver coil, the net amount of power loss increases with the increase of distance between the transmitter coil and the receiver coil.

The efficiency in case of both alternating electromagnetic field and discontinuous direct electromagnetic field has been presented in "Table 7". Efficiency has been calculated by using this equation,

$$
\eta=\frac{P_{\text {OUTPUT }}}{P_{\text {INPUT }}} \times 100 \%
$$

Here, $\eta=$ Efficiency.

Table 7. Efficiency of both cases at different distances.

\begin{tabular}{|c|c|c|}
\hline $\begin{array}{c}\text { Distance } \\
(\mathbf{c m})\end{array}$ & $\begin{array}{c}\text { Alternating } \\
\text { EM Field } \\
(\boldsymbol{\%})\end{array}$ & $\begin{array}{c}\text { DDEM Field } \\
(\mathbf{\%})\end{array}$ \\
\hline 0 & 99.77 & 71.51 \\
\hline 1 & 46.98 & 28.74 \\
\hline 2 & 16.25 & 9.94 \\
\hline 3 & 11.27 & 5.10 \\
\hline 4 & 10.59 & 2.42 \\
\hline 5 & 6.58 & 0.75 \\
\hline
\end{tabular}

The calculated data of "Table 7" has been plotted at "Fig. 10" which presents that, the efficiency in in case of both alternating electromagnetic field and discontinuous direct electromagnetic field decrease with the increase of distance between the transmitter coil and the receiver coil as well as the efficiency case of alternating electromagnetic field is

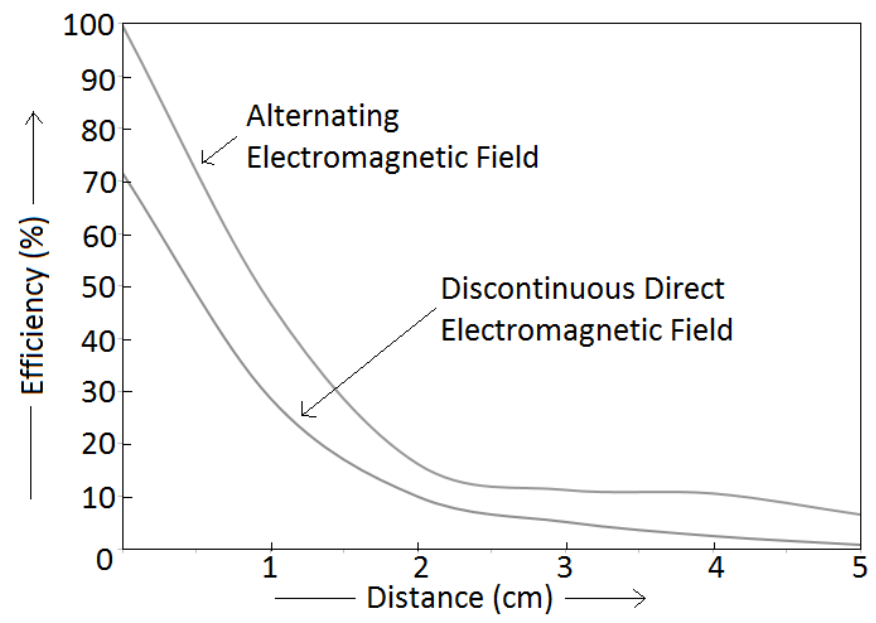

Fig. 10. Graphical representation of efficiency of both cases at different distances.

always higher than the efficiency in case of discontinuous direct electromagnetic field in between $0 \mathrm{~cm}$ to $5 \mathrm{~cm}$ distances between the transmitter coil and the receiver coil.

\section{CONCLUSION}

All the observations as well as calculations indicates that, in between alternating electromagnetic field and discontinuous direct electromagnetic field, the wirelessly received output voltage is higher in case of discontinuous direct electromagnetic field at high distance between the transmitter coil and the receiver coil. That means, the wireless power transmission range can be increased by using discontinuous direct electromagnetic field. The total amount of transferred power is also slightly higher in that case but the net amount of power loss is always higher in that case. As a result, the overall efficiency will decrease if the discontinuous direct electromagnetic field can be used for wireless power transmission. So, using the alternating magnetic field is the efficient way of wireless power transmission.

At the time of experiment, all the data in case of alternating electromagnetic field has been observed for a very high operating frequency as $127 \mathrm{kHz}$ and all the data in case of discontinuous direct electromagnetic field has been observed for a very low operating frequency as $10 \mathrm{~Hz}$, due to instrumental insufficiency. From the present observations, it can be assumed that, the wireless power transmission range will be increase with the increase of operating frequency.

\section{ACKNOWLEDGMENTS}

Special thanks to Mr. Ebad Zahir, Special Assistant, Department of EEE; Assistant professor, Faculty of Engineering, American International University-Bangladesh, for all of his inspirations and motivational words in different aspects of study life.

\section{REFERENCES}

[1] A.B.M. Khalid Hassan, Md. Rokibul Hassan Billah, and Md. Al Amin. 2015. Wireless Power Transfer Module Design using Discontinuous Direct Electromagnetic Field. International Journal of Computer Applications, Vol. 113, No. 5.

[2] Siqi Li, and Chunting Chris Mi. 2015. Wireless Power Transfer for Electric Vehicle Applications. IEEE Journal of Emerging and Selected Topics in Power Electronics, Vol. 3, No. 1. 